\title{
Postdoctoral Research Grants Writing: Moving A Step Ahead Ehtesham Arif
}

Renal Electrolytes and Hypertension Division, University of Pennsylvania, 3400 Civic Center Blvd, Philadelphia, PA 19104,USA

Email: ehtesham@mail.med.upenn.edu

\begin{abstract}
Availing the opportunity to get an independent research grant at the postdoctoral level not only provides encouragement and stability but also opens up new avenues of research and academic success. Awareness about research grants in terms of availability, eligibilities as well as a good research plan increases the chance of success. The present article is inspired by my research experiences in the United States. I tried to compile the sources for research grants, their eligibilities, and provide basic knowledge about how to design and write a proposal that will have the best chance for success. In my opinion this commentary will benefit the early stage researchers, especially non-immigrant researchers within the United States.
\end{abstract}

Keywords: academic career, postdoctoral grants, research funding

In the highly competitive world of research, obtaining a postdoctoral grant not only provides stability to the career of a postdoctoral researcher but also a great source of encouragement for performing research. Additionally, it provides a solid platform for a postdoctoral researcher to prepare for the next phase that involves submitting career development grant applications. After completing my doctoral thesis from the Institute of genomics and integrative biology, Delhi, India, I joined Indiana University, Indianapolis as a postdoctoral fellow under the mentorship of Dr. Deepak Nihalani. From the first day of my joining in the group he motivated me to write an independent research grant for postdoctoral fellowship. After I moved with him to the University of Pennsylvania (UPENN), Philadelphia, I started exploring options to write and submit research grant proposals to various national and international agencies. I was fortunate to associate myself with the Biomedical Postdoctoral Council at the UPENN, who was instrumental in providing me with the information about career advancement in science. Since I was holding a J1 visa, I was unable to submit the majority of postdoctoral grants especially the ones sponsored by the
National Institute of Health (NIH). However, I was able to find several agencies that fund postdoctoral grants regardless of the immigrant status of a postdoctoral researcher. Initially, I applied for the postdoctoral grant from American Heart Association and although my proposal was appreciated, due to severe competition I did not get a fundable score. Undeterred by this failure, I applied to at the NephCure foundation that recognizes my field of specialization and funds kidney related studies. My proposal was rated as one of the best proposals in my category and therefore, I was awarded a two year grant from the Nephcure. I am thankful to my mentors, especially Dr. Nihalani whose motivation and encouragement at every step of my career helped me advance to this level. This article is based on my experiences in the scientific field and I sincerely hope that new researchers will take important lessons from this experience. Presently I am working towards an independent research grant from the NephCure foundation, and would like to share my ongoing experiences with the research community. 
There are several important factors that lead to a successful postdoctoral research proposal, which are listed below.

1. Hypothesis: A strong research hypothesis is central to any proposal. The hypothesis should be within the framework of the proposal and should be testable under the given conditions. It should also be fully supported by the preliminary observations. Reviewers pay close attention to your data analysis and your overall plans of executing your hypothesis. If research hypothesis is not written within the conceptual framework with clear and crisp statements, it can lead to negative feedback from the reviewers $(1,2)$.

2. Objectives: This part of the research proposal should describe the overall plan for the study by prioritizing them in primary, secondary and if applicable tertiary aims. It should describe active strategies that will be used to accomplish goals of the study. It is critical that the specific aims should be designed to prove the key elements of your hypothesis (3).

3. Research Design: It is described as a conceptual structure within which research will be carried out. It is a blueprint of research proposal that consist of sample collections, execution of the experiments, measurement and analysis of data. The title for proposal should be concise yet reflect the important message in the proposal. An abstract should be a self-contained summary of the most important elements of the research proposal including research questions, rational for the study, hypothesis statement, and concise information about the research design and experimental procedure that appropriately reflects the key elements of the proposal. The introduction should provide enough background and specify the need to perform this study and clearly identifies the research problem. This part of the proposal should include an overall plan to answer every question or to test every hypothesis illustrated in the hypotheses section. Overall, the research study should be highly focused on appropriate justification for the proposed research plan (1-4).
4. Methodology: In this section the overall working plan including selection criteria, interventions, research instruments, experimental procedure, ethical issues, data collection and analysis should be included. This section should describe about the way that research question devised in the hypothesis is going to be answered. Anticipated error and biasness should be explained and how it will be controlled, minimized and addressed. Reviewers love to see fancy new approaches that will be used in the proposal (1).

5. Mentorship and Infrastructure: Mentorship is one of the core components of a postdoctoral grant. Reviewers judge whether the mentor/s has a sufficient level of expertise and experience to guide and provide necessary facilities to complete the research work. The parent institution and the infrastructure it offers where the study will be conducted also plays a critical role in the success of the proposal. It is also important to identify research collaborators in your field who will be instrumental in the successful execution of your proposal. All these elements should be clearly stated in your proposal that will convince reviewers that you have access to the best available research resources and the intellectual input, which is critical for the success of your study (1-3).

6. References: Often agencies require two to three reference letters from the people who can judge your capabilities. As a postdoc researcher you should choose your referees carefully who can appropriately judge your strengths and weaknesses. Ideally, reference letters should be from your mentors and the department chair of your university.

There are several finding agencies in the United States and Europe that support postdoctoral fellowship in their early phases of their careers. Although National Institute of Health (NIH) and National Science Foundation (NSF) provide grants on a regular basis to U.S Citizens, there are several other scientific organizations such American heart association (AHA), American 
Association for Cancer Research (AACR), NephCure foundation, Life sciences Research Foundation, Society in Science, Human Frontier Science program, Helen Hay Whitney Foundation and Fulbright Post-Doctoral Fellowships that provide opportunities to non-immigrant postdoctoral fellows working in accredited universities in the United States. Complete list of non-NIH postdoctoral grant are available elsewhere (5). Beside that non-immigrants are eligible for the advanced postdoctoral scientist award under NIH K99 program. It is critical to realize that according to $\mathrm{NIH}$ definition $(5,6)$ a fellow can remain as a postdoc for only five years and therefore, the planning and timing for a postdoctoral application is critical. Ideally, a fellow should start this process within the second year of postdoctoral fellowship when the chances of receiving a postdoctoral fellowship are higher. Obtaining a postdoctoral grant establishes a foundation for the next level of career development grants and helps in securing faculty position in academia.

\section{Acknowledgements}

NephCure Foundation (NCF), NephCure Postdoctoral Grants 2012-RFP-001 to E. A., is duly acknowledged. Dr. Deepak Nihalani is highly acknowledged for his encouragement and support for writing of this article.

\section{References}

1. Asya Al-Riyami, How to prepare a Research Proposal. Oman Med J. Apr 2008; 23(2): 66-69.

2. Research associateship program: http://sites.nationalacademies.org/PGA/RAP/PG A_052087

3. F Pajares. The elements of a proposal. http://www.uky.edu/ eushe2/Pajares/Elements OfaProposal.pdf

4. http://www.enterprisedevelopment.org/page/download?id=2133

5. http://www.fic.nih.gov/FUNDING/NONNIH/Page s/postdoctoral.aspx
6.

http://history.nih.gov/research/downloads/stett en_fellow_handbook.pdf 